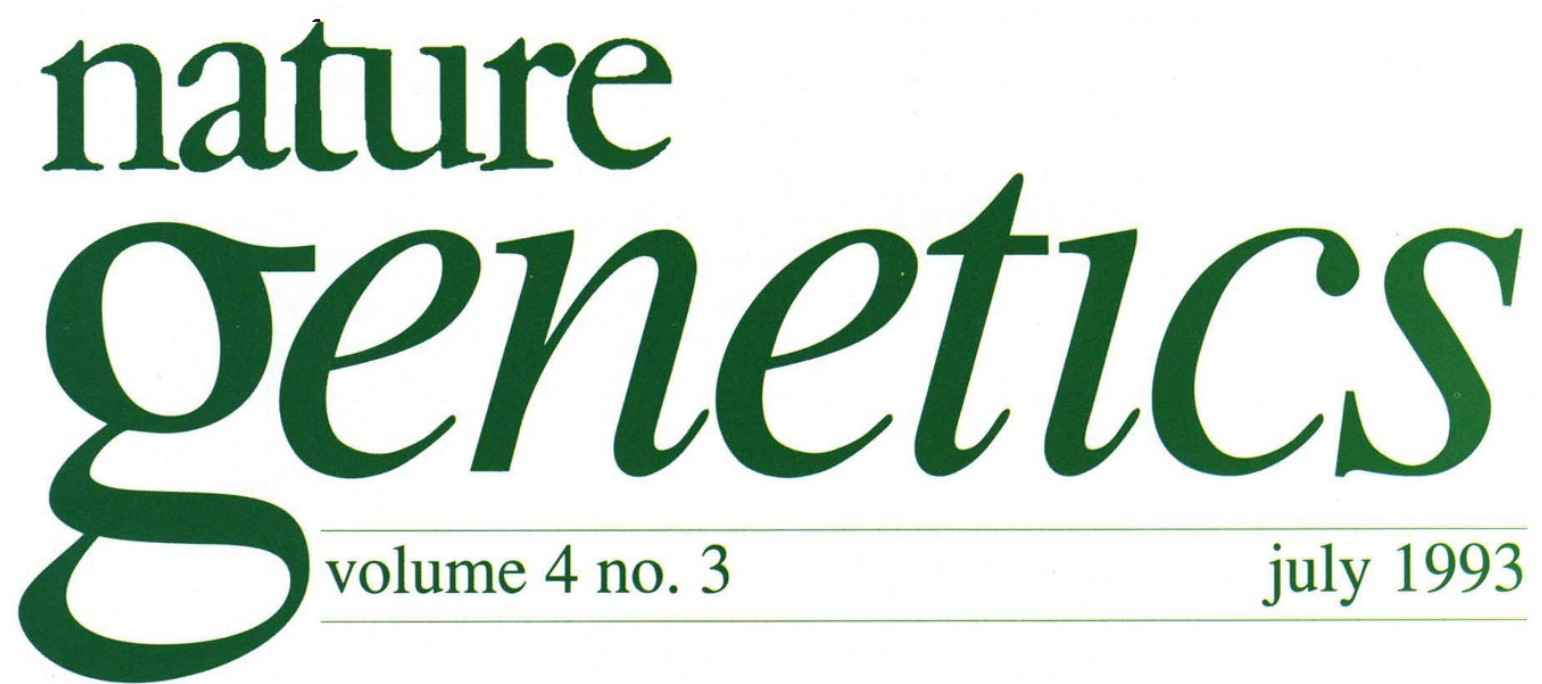

\title{
Diagnosing the heart of the problem
}

In the glamorous world of professional sports, few athletes are lucky enough to escape physical injury at some time or other. Debilitating ailments such as torn anterior cruciate ligaments and rotator cuffs are seemingly as commonplace in the sports pages as the anatomy textbooks. There is a growing awareness, however, that the strenuous physical exertion of competitive athletes carries its own potentially life-threatening risks. In the United States, heart failure, not physical injury, is the most common form of sudden death among young athletes: more than 100 athletes have died of sudden heart failure during the past 10 years, whereas only half that figure succumbed to severe physical trauma.

A relatively well-known cause of sudden death among athletes is Marfan syndrome, which claimed the life of volleyball player Flo Hyman. Marfan syndrome is characterized by excessive growth of limb bones (an obvious advantage for some sports) but also by a weakened aorta which is prone to rupture'. But perhaps the best known sports fatality in the United States was Hank Gathers, a talented 23-year-old college basketball player who collapsed from heart failure during a game on March 3, 1990 , and died hours later. Gathers suffered from hypertrophic cardiomyopathy, which affects 1 in 5,000 people and is characterized by increased thickening of the heart muscle and a life-threatening arrhythmia. Gathers had fainted on court three months earlier, but his subsequent medication (inderal) left him feeling lethargic, and he had taken a reduced dose the night he died.

Lately, two widely reported events have thrust the problem of hypertrophic cardiomyopathyback into the spotlight. Last year, the teenage son of Terry Yorath, a former Welsh international soccer player and the current team manager, suddenly collapsed and died while playing soccer at home. And just three months ago, on April 29, Reggie Lewis, the 27-year-old captain of the Boston Celtics, collapsed onto the parquet floor of the Boston Garden during a crucial basketball playoff game. Initial tests by the team physician failed to discern a serious problem, but when Lewis returned to the game he complained of further dizzy spells. Later in hospital, the team doctor assembled what he called a 'Dream Team' of 12 eminent cardiologists who proclaimed that Lewis suffered from hypertrophic cardiomyopathy, invoking instant memories of Gathers. Not surprisingly, the front page of The Boston Globe declared: "Lewis' career called probably over".

But the saga then took a bizarre twist, illustrating the severe difficulty of clinical diagnosis for this problem. Reportedly upset by the lack of direct consultation with his cardiologists, Lewis transferred to the Brigham and Women's Hospital for a second opinion. After extensive tests, it was announced that Lewis actually had a much more benign, neurological condition, neurocardiogenic syncope (sudden loss of consciousness brought about by abnormal signals from the vagus nerve to the heart), which can easily be managed with $\beta$ blockers. This left some highly distinguished cardiologists bickering in public about the accuracy of Lewis' diagnosis, with one member of the original group of physicians openly sceptical about the milder diagnosis ${ }^{2}$.

Lewis' plight has prompted officials in Boston 
and other US cities to advocate screening programs for young athletes to pinpoint potential cases at risk of sudden cardiac failure. (Some universities do screen their student athletes for Marfan syndrome.) In the United Kingdom, a pilot study is underway to detect warning signs in teenage athletes using echocardiography, but there are doubts that screening will be either feasible or effective $^{3}$, in part because healthy athletes frequently have hypertrophic hearts anyway.

Back to the bench: With time, an accurate molecular diagnosis of hypertrophic cardiomyopathy may become practical, but there are considerable hurdles to overcome, not least of which is the heterogeneity of the condition. However, there have been some encouraging advances in unravelling the molecular basis of hypertrophic cardiomyopathy of late, allowing the field to recover from an ignominious beginning. More than $50 \%$ of hypertrophic cardiomyopathy cases are known to be familial, inherited as an autosomal dominant trait, and most of the others are likely to be sporadic forms. In 1979, John Darsee and colleagues reported that familial hypertrophic cardiomyopathy (FHC) was linked to the HLA locus, only to retract that paper (among many others) four years later after admitting the data had been fabricated. It was not until 1989 that the principal FHC locus was mapped to chromosome 14. Studies from the group of J.G. and Christine Seidman at the Harvard Medical School have elegantly demonstrated that the common form of FHC is caused by a number of mutational mechanisms involving the $\beta$-cardiac myosin heavy chain gen $e^{4-6}$, which encodes one of the major contractile proteins of the cardiac myocyte. These mutations include unequal crossing over (with the adjacent $\alpha$-heavy chain gene), gene deletions and a host of missense mutations clustered in the globular head of the myosin polypeptide. Unequal crossing-over initially provided an attractive explanation for the abundance of sporadic hypertrophic cardiomyopathy cases, but the original family presenting with the hybrid myosin gene has subsequently been found to contain a deleterious missense mutation ${ }^{6}$.

There are at least two other loci for FHC that have been identified, as reported in Nature Genetics. In the first linkage study ${ }^{7}$, a new locus for what seems to be a severe form of FHC was assigned by the Harvard group to chromosome 1q. A cluster of candidate genes fall within this region, including tropomyosin, troponin I and actin, but the exact gene remains a mystery for now. At least half-a- dozen families with FHC did not show linkage to chromosome 1, suggesting even greater genetic heterogeneity. In this issue ${ }^{8}$, Ketty Schwartz and coworkers describe a third FHC locus on chromosome 11, having first ruled out a number of candidate genes. Other loci for FHC may still be found.

Progress is also being made in another congenital heart disorder associated with ventricular hypertrophy and sudden death (and which was raised at one time in connection with Lewis' condition) - Long QT syndrome (LQT) ${ }^{9}$. The discovery two years ago by Mark Keating's group that the locus for this form of ventricular arrhythmia was tightly linked to the Harvey ras-1 gene on chromosome $11 \mathrm{p}$ prompted speculation that the defective gene was an oncogene (ras can act as a regulator of cardiac ion channels). However, this intriguing notion has since been dismissed and researchers are down to a few hundred kilobases of DNA in their search for the gene. New data indicate that there is also a second locus for $\mathrm{LQT}^{10}$, as some families do not show linkage to chromosome 11 . There is one curious difference, however: deaths in these non-linked families are reported to occur in subjects at rest, rather than during periods of intense exercise or excitement.

Eventually, the molecular insights into the multiple congenital causes of heart disease will facilitate the diagnosis of these conditions. But the complexity of the $\beta$-MHC gene, which contains 40 exons, does not make screening of the sole known cause of FHC straightforward, even though such tests are being offered for families at risk in Britain and elsewhere. The effectiveness of treatment of the disease with drugs such as amiodarone is also uncertain ${ }^{3}$, although Reggie Lewis can take heart (no pun intended) that at least one professional basketball player with cardiomyopathy has played for ten years while taking medication. Nevertheless, Celtics fans will be hoping that the 'Dream Team's' bleak prognosis will suffer the same fate as that of their former franchise player, Larry Bird - an early retirement.

\section{References}

1. McKusick, V. Nature 352, 279-281 (1993).

2. Johnson, W.O. Sports Illustr. May 24, 36-41 (1993).

3. Clark, A.L. \& Coats, A.J.S. Br. med. J. 306, $409-410$ (1993).

4. Tanigawa, G. et al. Cell 62, 991-998 (1990).

5. Geisterfer-Lowrance, A.A.T. et al. Cell 62, 999-1006 (1990).

6. Watkins, H. et al. Now Engl. J. Med. 326, 1108-1114 (1992).

7. Watkins, H. et al. Nature Genet. 3, 333-337 (1993).

8. Carrier, L. et al. Nature Genet. 4, 311-313 (1993).

9. Keating, M. et al. Science 252, 704-706 (1991).

10. Curran, M. et al. J. clin. Invest. (in the press). 